\title{
ANALYTICAL AND PRACTICAL STUDY OF COUPLED MULTIVIBRATORS
}

\author{
UMESH KUMAR \\ Senior Member, IEEE; Fellow, IETE \\ P. BHUSHAN MITAL \\ Fellow, IETE
}

This paper investigates the dynamic behavior of a system of two multivibrators. It is shown that the characteristics of synchronization states of multivibrators are coupled in a particular manner and their approach to synchronization can be tackled analytically without approximations. This can be regarded as an advancement, since most of the analytical work on coupled oscillators attain results only by resorting to an approximate mathematical method.

It has been shown that the results obtained for coupled multivibrators are similar to those of sinusiodal oscillators.

Such systems are important in the fields of theoretical biology, communication, radar system technology, and coupled solar cells.

\section{BASIC MULTIVIBRATOR INVOLVED IN THE SYSTEM}

\section{1) The Basic Triangular/Square Wave Generator}

The block diagram in Fig. 1 represents a relatively general class of various triangular-square wave generator that possess in common a hysteretic comparator and integrator in a closed loop.

\section{ASTABLE MULTIVIBRATOR}

Both integration and hysteretic comparisons can be performed by employing merely one operational amplifier, Fig. 2. The triangular wave here is not linear, as a result of the simple resistor capacitor integrator used in this circuit.

The sides of triangular waveshape are exponentials. The linearity can be improved by reducing the ratio $R_{a} / R_{b}$. The frequency of oscillation is given by

$$
f=\frac{1}{2 R C \ln (1+2 K)} ; K=R_{a} / R_{b}
$$

\section{PRECISE TRIANGULAR WAVE GENERATOR}

To linearize the triangles, it is required that $\mathrm{C}$ be charged with a constant current rather than the exponential current supplied through $R$. 


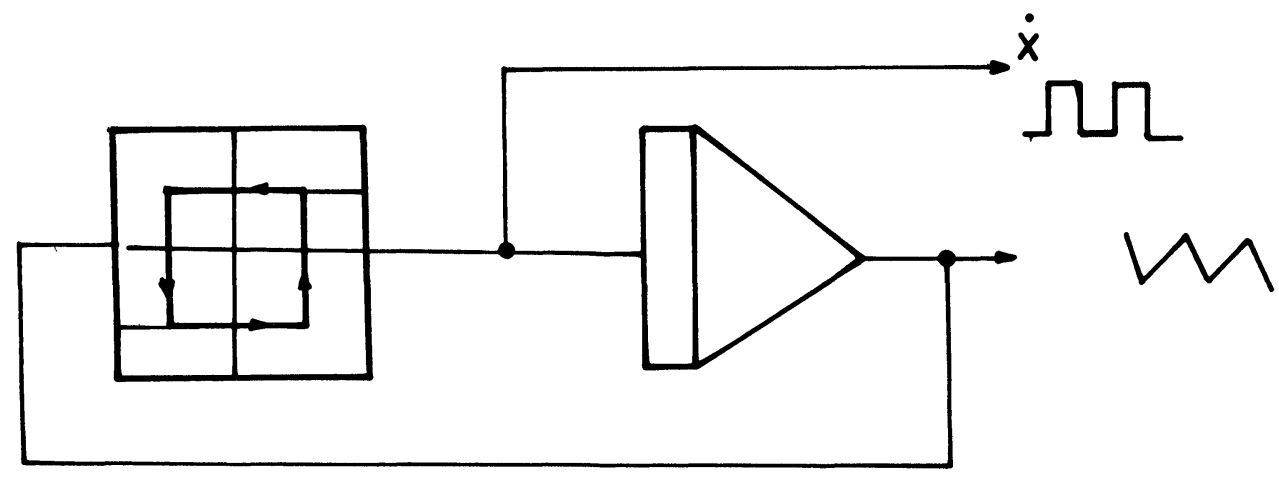

FIGURE 1

To get a precise triangular wave, we employ a second operational amplifier to perform integration so that a constant supply current to $\mathrm{C}$ is obtained.

$f=\frac{1}{4 K R C} ; K=\frac{R_{a}}{R_{b}}$

Dynamic equation.

$\dot{\mathrm{X}}=\mathrm{V}_{\mathrm{Z}} \operatorname{Sign}\left(\mathrm{K} \dot{\mathrm{X}}-\frac{1}{\mathrm{RC}} \mathrm{X}\right)$

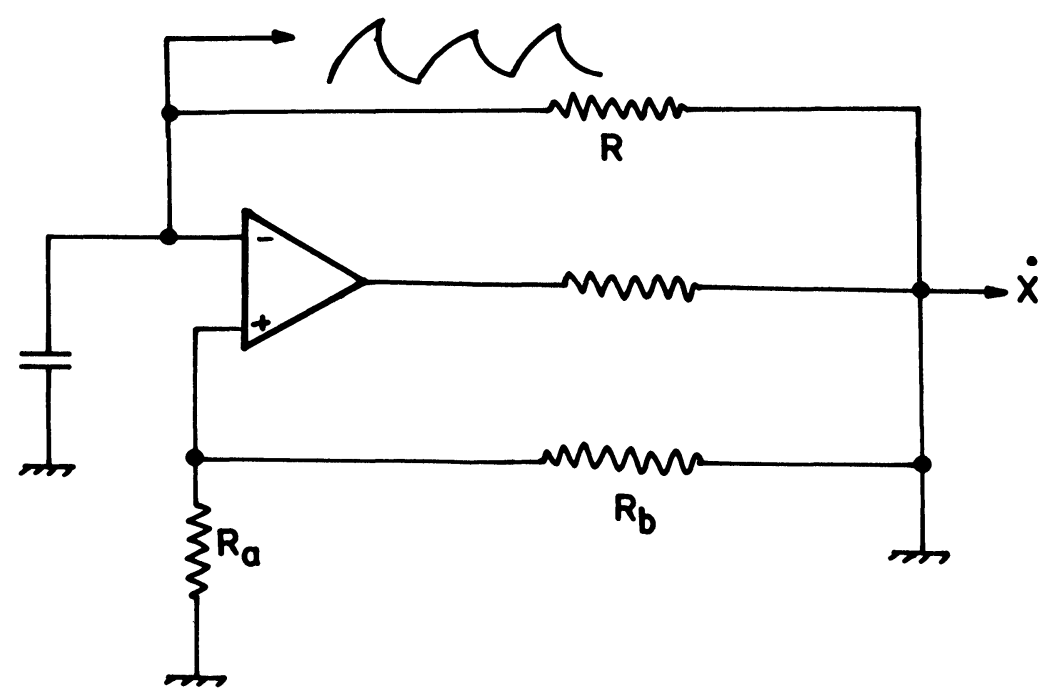

几几

FIGURE 2 


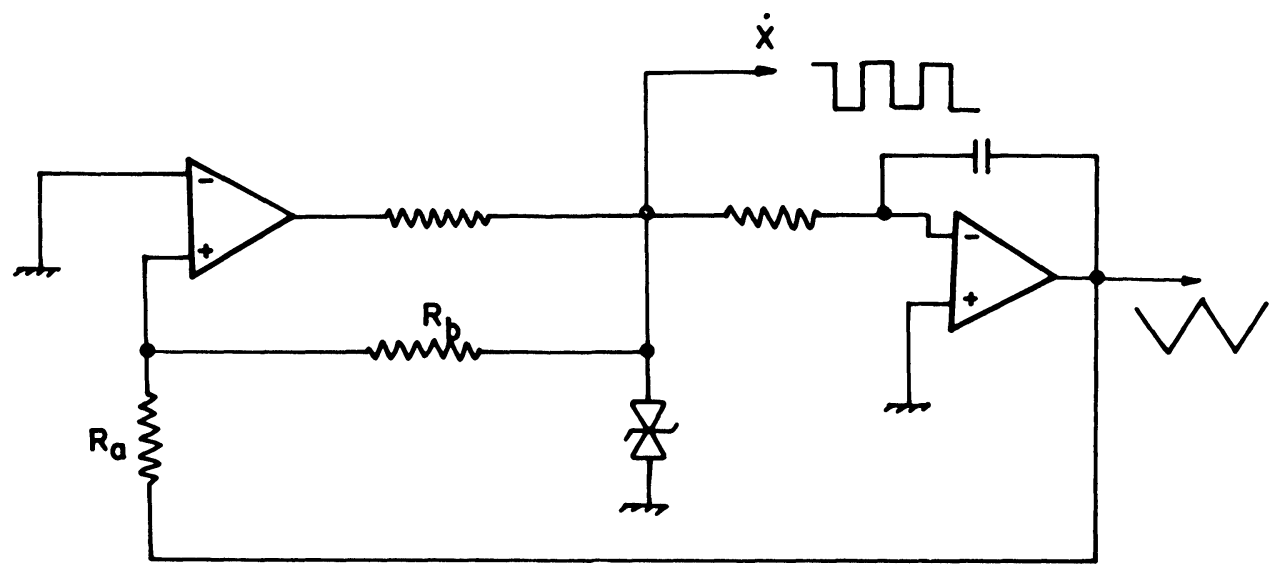

FIGURE 3

Where Sign $U= \begin{cases}+1 & \text { for } U \geqslant 0 \\ -1 & \text { for } U<0\end{cases}$

$$
\operatorname{Sign}(U) \neq 0 \text { for } U=0
$$

$\dot{\mathrm{X}}=\mathrm{V}_{\mathrm{Z}} \operatorname{Sign}(\dot{\mathrm{X}}-4 \mathrm{fx})$

\section{TWO MUTUALLY COUPLED MULTIVIBRATORS}

It is assumed that the two oscillators possess equal square wave amplitudes and equal triangular wave amplitudes, namely

$\mathrm{V}_{\mathrm{Z}}=\mathrm{V}_{\mathrm{zZ}}=\mathrm{V}_{\mathrm{Z}} ;$ also $\mathrm{K}_{1}=\mathrm{K}_{2}=\mathrm{K}$

The oscillators have been coupled by letting each oscillator's oscillator integrator integrate not only its own wave, but also the other oscillator square wave with a different time constant. This is shown in Fig. 4.

Let $\dot{X}_{1}$ and $\dot{X}_{2}$ be the comparators output values, then the integrate output $Y_{1}$ and $Y_{2}$ are given by

$$
\begin{aligned}
& Y_{1}=-\left(\frac{X_{1}}{J_{1}}+\frac{X_{2}}{J_{12}}\right) \\
& Y_{2}=-\left(\frac{X_{2}}{J_{2}}+\frac{X_{1}}{J_{21}}\right)
\end{aligned}
$$

Where $J_{1}=R_{1} C_{1}$

$$
J_{12}=R_{12} C_{1}
$$




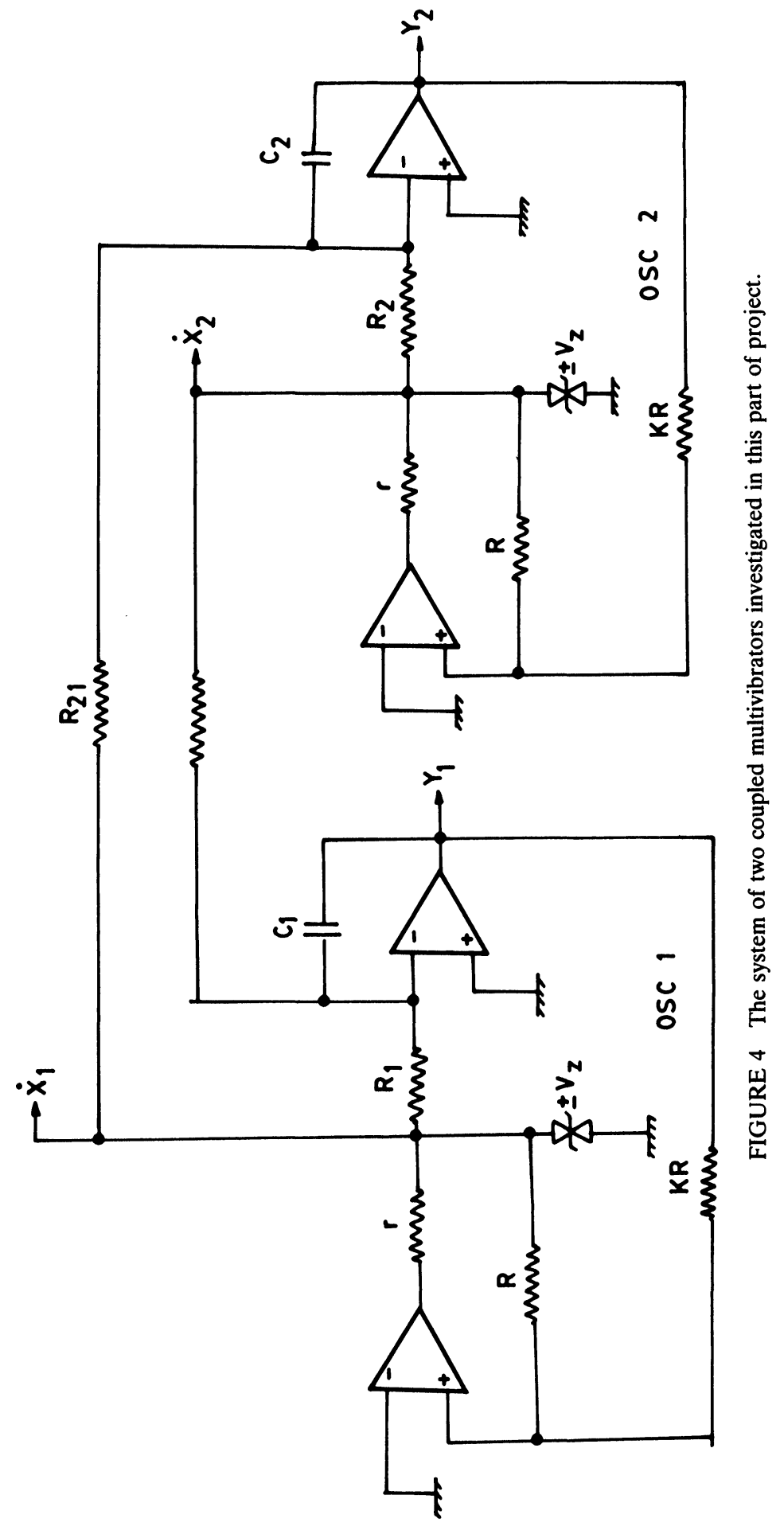




$$
\begin{aligned}
\mathbf{J}_{2} & =\mathrm{R}_{2} \mathrm{C}_{2} \\
\mathbf{J}_{21} & =\mathrm{R}_{21} \mathrm{C}_{2}
\end{aligned}
$$

Although $Y_{1}$ and $Y_{2}$ are no longer triangular waves, they preserve their previous (uncoupled) amplitude. This is due to the fact that the trip points are unchanged.

Using (3) and (4), the dynamic equations of the coupled configuration can be written as

$$
\begin{aligned}
& \dot{X}_{1}=V_{Z} \operatorname{Sign}\left[\dot{X}_{1}-4 \dot{f}_{1}\left(X_{1}+\frac{T_{1}}{T_{2}} X_{2}\right)\right] \\
& \dot{X}_{2}=V_{Z} \operatorname{Sign}\left[\dot{X}_{2}-4 \dot{f}_{2}\left(X_{2}+\frac{T_{2}}{T_{21}} X_{1}\right)\right]
\end{aligned}
$$

Where $\dot{\mathrm{f}}_{1}$ and $\dot{\mathrm{f}}_{2}$ are the intrinsic (uncoupled) frequencies of oscillators, namely

$$
\dot{\mathrm{f}}_{1}=\frac{1}{4 \mathrm{KT}} ; \dot{\mathrm{f}}_{2}=\frac{1}{4 \mathrm{KT}_{2}}
$$

\section{DYNAMIC BEHAVIOR OF MUTUALLY COUPLED OSCILLATORS}

The main objective of this section is to evaluate the frequency of oscillations and the phase shift between the two oscillations. It is assumed that the coupled oscillators oscillate with periods $T_{i}^{1}$ and $T_{i}^{2}$, where subscript $i$ denotes the ith half cycle of oscillation in Fig. 5a. Let the square wave $\dot{X}_{2} \operatorname{lag} \dot{X}_{1}$ by $\hat{\mathbf{J}}_{i}$, i.e.,

$$
\frac{-\mathbf{J}_{\mathrm{i}}^{1}}{2}<\hat{\mathbf{J}}_{\mathrm{i}}<0
$$

For the lagging situation $\hat{\mathrm{J}}_{i}$ is assumed to possess a negative value while for a leading situation it possess a positive value.

The output value of the second oscillator comparator will change at the end of its ith half cycle from $\pm V_{Z}$ to $\mp V_{Z}$. It is possible to evaluate the half cycle length by equating this transitional change of $2 \mathrm{~V}_{\mathrm{Z}}$ at the comparator end to the corresponding integrator voltage that causes such transition. It can be shown that, when $\dot{\mathrm{X}}_{2}$ lags $\dot{\mathrm{X}}_{1}$ (Fig. 5a):

$$
t_{B}-t_{A}=\frac{1}{2} T_{i}^{1}-\left|\hat{J}_{i}\right|
$$




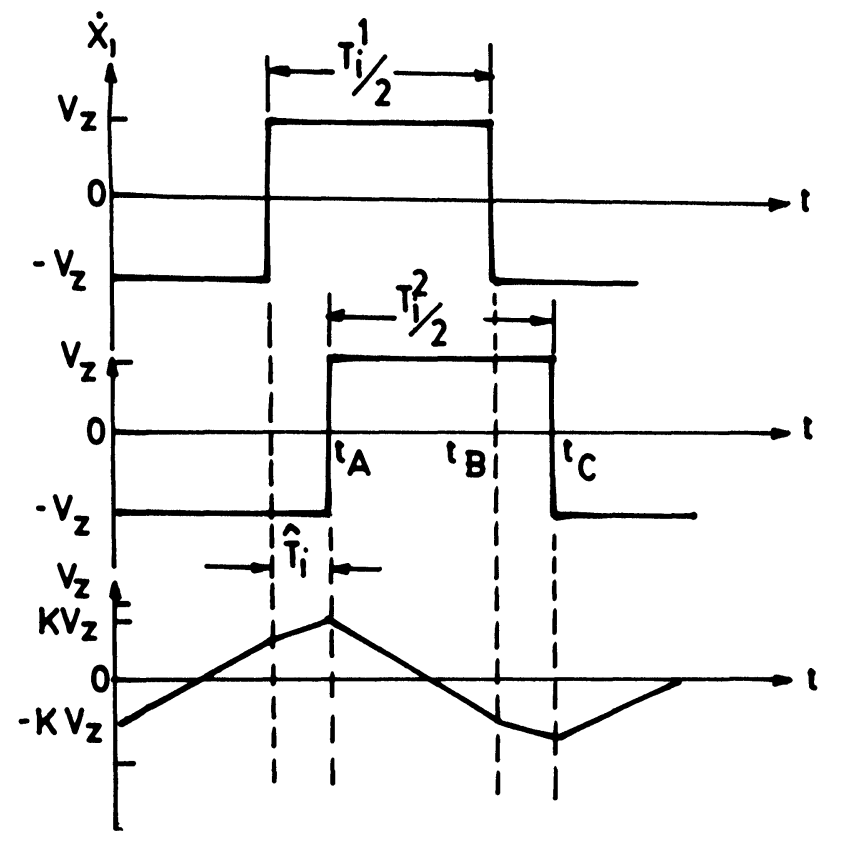

OSC 1

OSC 2

INTEG 2

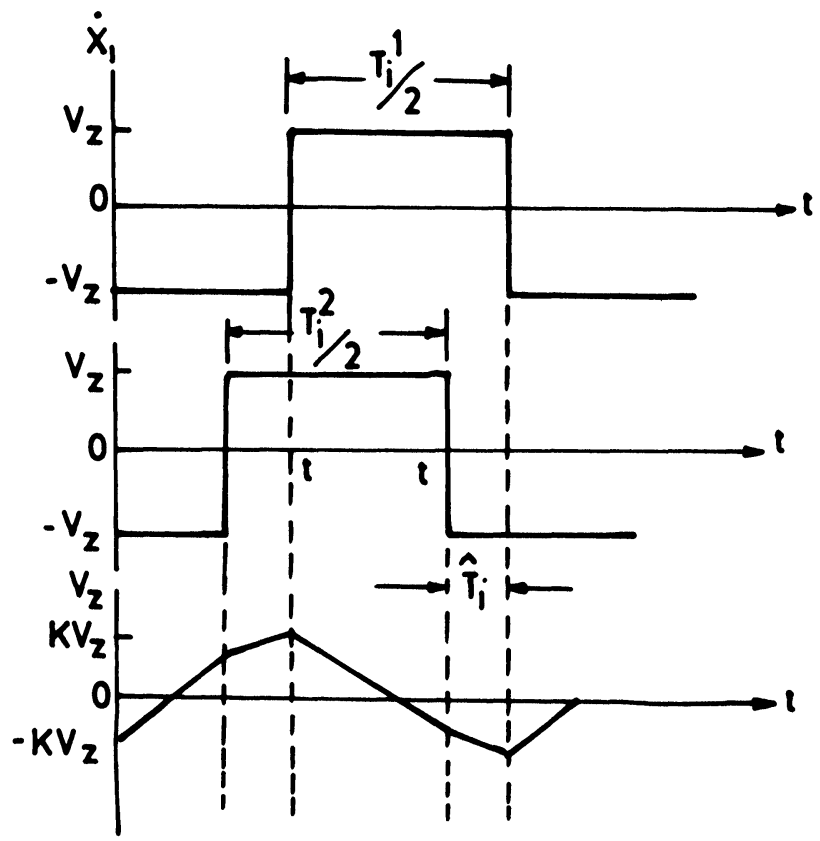

OSC 1

OSC 2

INTEG 2

FIGURE 5 The mutual positions (in time) of the waves generated by the two coupled multivibrators. 
and

$$
\begin{aligned}
t_{C}-t_{B} & =\frac{1}{2} T_{i}^{2}+\left|\hat{J}_{i}\right|-\frac{1}{2} T_{i}^{1} \\
& =\frac{1}{2} D T_{i}+\left|\hat{J}_{i}\right|
\end{aligned}
$$

where

$\mathrm{DT}_{\mathrm{i}}=\mathrm{T}_{\mathrm{i}}^{2}-\mathrm{T}_{\mathrm{i}}^{1}$

Due to the ratio $\mathrm{K}$ between the resistors values at the comparator positive input terminal, a voltage transition of $2 \mathrm{~V}_{\mathrm{Z}}$ at the comparator is equivalent to a voltage change of $2 \mathrm{KV}_{\mathrm{Z}}$ at the integrator output. As a result,

$$
\left(t_{B}-t_{A}\right)\left[\frac{V_{Z}}{J_{2}}+\frac{V_{Z}}{J_{21}}\right]+\left(t_{C}-t_{B}\right)\left[\frac{V_{Z}}{J_{2}}-\frac{V_{Z}}{J_{21}}\right]=2 V_{Z} K
$$

Dividing both sides of (6) by $V$ and putting the value of $\left(t_{B}-t_{A}\right)$ and $\left(t_{C}-t_{B}\right)$ from equations (a) and (b) we get,

$$
\left(\frac{1}{2} \mathrm{~T}_{\mathrm{i}}^{1}+\hat{\mathbf{J}}_{\mathrm{i}}\right)\left[\frac{1}{\mathrm{~J}_{2}}+\frac{1}{\mathrm{~J}_{21}}\right]+\left(\frac{1}{2} \mathrm{DT}_{\mathrm{i}}+\hat{\mathbf{J}}_{1}\right)\left[\frac{1}{\mathrm{~J}_{2}}-\frac{1}{\mathrm{~J}_{21}}\right]=2 \mathrm{~K}
$$

Multiplying both sides of equation (7) by 2 and rearranging we get

$$
\begin{aligned}
& \left(\mathrm{J}_{\mathrm{i}}^{1}+2 \hat{\mathrm{J}}_{\mathrm{i}}\right)\left[\frac{\mathrm{J}_{21}}{\mathrm{~J}_{2}}+1\right]+\left(\mathrm{DT}_{\mathrm{i}}-2 \hat{\mathrm{J}}_{\mathrm{i}}\right)\left[\frac{\mathrm{J}_{21}}{\mathrm{~J}_{2}}-1\right]=4 \mathrm{~K} \mathrm{~J}_{21} \\
& \Longrightarrow \mathrm{DT}_{\mathrm{i}}\left(\frac{\mathbf{J}_{21}}{\mathbf{J}_{2}}-1\right)=4 \mathrm{~K} \mathrm{~J}_{21}-\mathrm{T}_{\mathrm{i}}^{1}\left(\frac{\mathbf{J}_{21}}{\mathrm{~J}_{2}}+1\right)-4 \hat{\mathbf{J}}_{\mathrm{i}}
\end{aligned}
$$

Similar equations can be written for the case when $\dot{\mathrm{X}}_{2}$ leads $\hat{\mathrm{X}}_{1}$, i.e., $0 \leqslant \hat{\mathrm{J}}_{\mathrm{i}} \leqslant$ $\mathbf{J} \mid / 2$.

Referring to Fig. (5b),

$$
\begin{aligned}
& t_{B}-t_{A}=\frac{1}{2} D T_{i}+\left|\hat{J}_{i}\right| \\
& t_{C}-t_{B}=T_{i}^{1} / 2-\hat{J}_{i}
\end{aligned}
$$

from the logic given before, we get in this case,

$$
\left(t_{B}-t_{A}\right)\left[\frac{V_{Z}}{J_{2}}-\frac{V_{Z}}{T_{21}}\right]+\left(t_{C}-t_{B}\right)\left[\frac{V_{Z}}{J_{2}}+\frac{V_{Z}}{J_{21}}\right]=2 K V_{Z}
$$


Using equations (a) and (b) and multiplying by $\mathrm{V}_{\mathrm{Z}}$

$$
\left(\frac{1}{2} \mathrm{DT}_{\mathrm{i}}+\left|\hat{\mathbf{J}}_{\mathrm{i}}\right|\right)\left[\frac{1}{\mathrm{~J}_{2}}-\frac{1}{\mathbf{J}_{21}}\right]+\left(\frac{1}{2} \mathrm{DT}_{\mathrm{i}}+\hat{\mathbf{J}}_{\mathrm{i}}\right)\left[\frac{1}{\mathbf{J}_{2}}-\frac{1}{\mathbf{J}_{21}}\right]=2 \mathrm{~K}
$$

Multiplying (7a) by 2 and rearranging we get,

$$
\mathrm{DT}_{\mathrm{i}}\left(\frac{\mathrm{J}_{21}}{\mathbf{J}_{2}}+1\right)=4 \mathrm{~K} \mathrm{~J}_{21}-\mathrm{T}_{\mathrm{i}}^{1}\left(\frac{\mathrm{J}_{21}}{\mathrm{~J}_{2}}+1\right)+4 \mathrm{~J}_{\mathrm{i}}
$$

The two equations (8) and (9), (one for the lagging case and the other for the leading case) can now be lumped together, using the 'Sign' function. This yields

$\operatorname{DT}_{i}\left[\frac{\mathbf{J}_{21}}{J_{2}} \operatorname{Sign}\left(\hat{\mathbf{J}}_{\mathrm{i}}\right)+1\right]=\left[4 \mathrm{~K} \mathrm{~J}_{21}-\mathrm{T}_{\mathrm{i}}^{\mathbf{l}}\left(\frac{\mathbf{J}_{21}}{\mathbf{J}_{2}}+1\right)\right] \operatorname{Sign}\left(\hat{\mathbf{J}}_{\mathrm{i}}\right)+4 \hat{\mathbf{J}}_{\mathrm{i}}$

where $\left|\hat{J}_{i}\right| \leqslant T_{i}^{1} / 2$

Those equations represent the integrator output of second multivibrator.

Similar analysis for the second multivibrator (considering both lagging and leading cases) will lead to a corresponding relationship, namely,

$\operatorname{DT}_{\mathrm{i}}\left[\frac{\mathrm{J}_{12}}{\mathrm{~J}_{1}} \operatorname{Sign}\left(\hat{\mathrm{J}}_{\mathrm{i}}\right)-1\right]=-\left[4 \mathrm{~K} \mathrm{~J}_{12}-\mathrm{T}_{\mathrm{i}}^{2}\left(\frac{\mathrm{J}_{12}}{\mathrm{~J}_{1}}+1\right)\right] \operatorname{Sign}\left(\hat{\mathrm{J}}_{\mathrm{i}}\right)-4 \hat{\mathbf{J}}_{1}$

Now we add equations (10) and (11) and, hence, eliminate $\hat{\mathrm{J}}_{\mathrm{i}}$. This yields

$\mathrm{DT}_{\mathrm{i}}\left(\frac{\mathrm{J}_{21}}{\mathrm{~J}_{2}}-1\right)=4 \mathrm{~K}\left(\mathrm{~J}_{21}-\mathrm{J}_{12}\right)-\mathrm{T}_{\mathrm{i}}^{1}\left(\frac{\mathrm{J}_{21}}{\mathrm{~J}_{2}}-\frac{\mathrm{J}_{12}}{\mathrm{~J}_{1}}\right)$

The above equation is for the ith half cycle.

One can write an equation similar to $(10)$ for the $(i+1)$ th half cycle. If $(10)$ is then subtracted from the similar equation, one would obtain

$\operatorname{DT}_{\mathrm{i}}\left[\frac{\mathrm{J}_{21}}{\mathbf{J}_{2}} \operatorname{Sign}\left(\hat{\mathrm{J}}_{\mathrm{i}}\right)+1\right]=-\mathrm{DT}_{\mathrm{i}}-\Delta \mathrm{T}_{\mathrm{i}}^{1}\left[\frac{\mathrm{J}_{21}}{\mathbf{J}_{2}}+1\right] \operatorname{Sign}\left(\hat{\mathrm{J}}_{\mathrm{i}}\right)$

where $\Delta D T_{i}=D T_{i+1}-D T_{i}$

and $\Delta T_{i}^{1}=T_{i+1}^{1}-T_{i}^{1}$

Equation (12) assists in substituting for $\mathrm{DT}_{\mathrm{i}}$ in equation (9) by

$$
D T_{i}=\frac{4 K\left(J_{21}-J_{12}\right)-J_{i}\left(\frac{J_{21}}{J_{2}}-\frac{J_{12}}{J_{1}}\right)}{\frac{J_{21}}{J_{2}}-1}
$$


Now substituting values in equation (13)

$$
\left\{1+\left[\frac{1-\frac{J_{12} \cdot J_{21}}{J_{1} J_{2}}}{\frac{J_{21}}{J_{2}}-\frac{J_{12}}{J_{1}}}\right] \operatorname{Sign}\left(\hat{J}_{i}\right)\right\} \Delta T_{i}^{1}+T_{i}^{1}=\left\{\frac{4 K\left(J_{21}-J_{12}\right)}{\frac{J_{21}}{J_{2}}-\frac{J_{12}}{J_{1}}}\right\}
$$

The following difference equation is obtained

$$
A \Delta T_{i}^{1}+T_{i}^{1}=T
$$

where

$$
A=1+\left[\frac{1-\frac{\mathbf{J}_{12} \mathbf{J}_{21}}{\mathbf{J}_{1} \mathbf{J}_{2}}}{\frac{\mathbf{J}_{21}}{\mathbf{J}_{2}}-\frac{\mathbf{J}_{12}}{\mathbf{J}_{1}}}\right] \operatorname{Sign}\left(\hat{\mathbf{J}}_{\mathrm{i}}\right)
$$

and

$T=\frac{4 K\left(J_{21}-J_{12}\right)}{J_{21} / J_{2}-J_{12} / J_{1}}$

To find the solution of the difference equation (15), we proceed in the following manner. Let the value of $i=0$

$$
\begin{aligned}
\left(J_{i}^{1}-J_{0}^{1}\right) A+T_{0}^{1} & =T \\
\Longrightarrow T_{0}^{1}+A T_{i}^{1}-T_{0}^{1} A & =T
\end{aligned}
$$

This gives an expression for $T_{i}^{1}$ in terms of $T$ and $A$

$$
T_{i}^{1}=\frac{T+T_{0}^{1}(A-1)}{A}=\frac{T}{A}+\frac{T_{0}^{1}}{A}(A-1)
$$

Similarly we find an expression for $T_{2}^{1}$

$$
\begin{aligned}
T_{2}^{1} & =T / A+\frac{T_{i}^{1}(A-1)}{A}=T / A+\frac{(A-1)}{A}\left[T / A+T_{0}^{1} \frac{(A-1)}{A}\right] \\
& =T_{0}^{1} \frac{(A-1)^{2}}{A}+T / A+T / A \frac{(A-1)}{A} \\
& =T_{0}^{1} \frac{(A-1)^{2}}{A}+T\left[\left(\frac{1}{A}+\frac{(A-1)}{A^{2}}\right)\right]
\end{aligned}
$$


Expression for the ith cycle

$$
\begin{aligned}
T_{i}^{1}= & T_{o}^{1} \frac{(A-1)^{2}}{A} \\
& +T\left(\frac{1}{A}+\frac{(A-1)}{A^{2}}+\frac{(A-1)^{2}}{A^{3}}+\cdots+\frac{(A-1)^{i-2}}{A^{i-1}}+\frac{(A-1)^{i-1}}{A^{i}}\right)
\end{aligned}
$$

as $\mathrm{i} \rightarrow \infty$, the 2 nd term reduces to unity, since the sum of such a series $\mathrm{S}$ is

$$
\sum S-\left[\frac{a}{1-r}\right]=\frac{1 / A}{1-\frac{(A-1)}{A}}=1 \quad \begin{aligned}
& \text { where } a=\text { first term } \\
& \text { and } r=\text { common factor }
\end{aligned}
$$

So the equation $(\mathrm{C})$ becomes

$$
T_{i}^{1}=T_{o}^{1}\left(\frac{A-1}{A}\right)^{i}+T
$$

Now for synchronization steady state we need that ith and $(i+1)$ th half period be of the same duration, i.e.,

$\Delta \mathrm{T}_{\mathrm{i}}^{1}=0 \Longrightarrow \mathrm{DT} \mathrm{T}_{\mathrm{i}}^{1}=0=\mathrm{T}_{\mathrm{i}}^{2}-\mathrm{T}_{\mathrm{i}}^{1} \Longrightarrow \mathrm{T}_{\mathrm{i}}^{1}=\mathrm{T}_{\mathrm{i}}^{2}$

so that in equation (19) $T_{i}^{1} \ldots$ becomes independent of $i$. This requires that the first term in the equation (19) should reduce to zero and $T$ should be greater than zero.

Depending on whether $A$ is larger or less than $1 / 2$, the absolute value of the transient response $\mathrm{T}_{\mathrm{o}}^{\mathrm{l}}(\mathrm{A}-1 / \mathrm{A})^{\mathrm{i}}$ will approach zero or will increase respectively when the number of steps $i$ increases. $A$ condition for $A$ being larger than $1 / 2$ is (16)

$$
\left[\frac{1-\frac{\mathbf{J}_{12} \mathbf{J}_{21}}{\mathbf{J}_{2} \mathbf{J}_{1}}}{\frac{\mathbf{J}_{21}}{\mathbf{J}_{2}} \frac{\mathbf{J}_{12}}{\mathbf{J}_{1}}}\right] \operatorname{Sign}\left(\hat{\mathbf{J}}_{\mathrm{i}}\right)>-1 / 2
$$

Let

$Q=\left(1-\frac{J_{12} J_{21}}{J_{2} J_{1}}\right) /\left(\frac{J_{21}}{J_{2}}-\frac{J_{12}}{J_{1}}\right)$

then equation $(20)$ reduces to

$Q$ Sign $\hat{\mathbf{J}}_{\mathrm{i}}>-1 / 2$

Now there are two possibilities of Sign $\hat{\mathbf{J}}_{\mathbf{i}}= \pm 1$. 


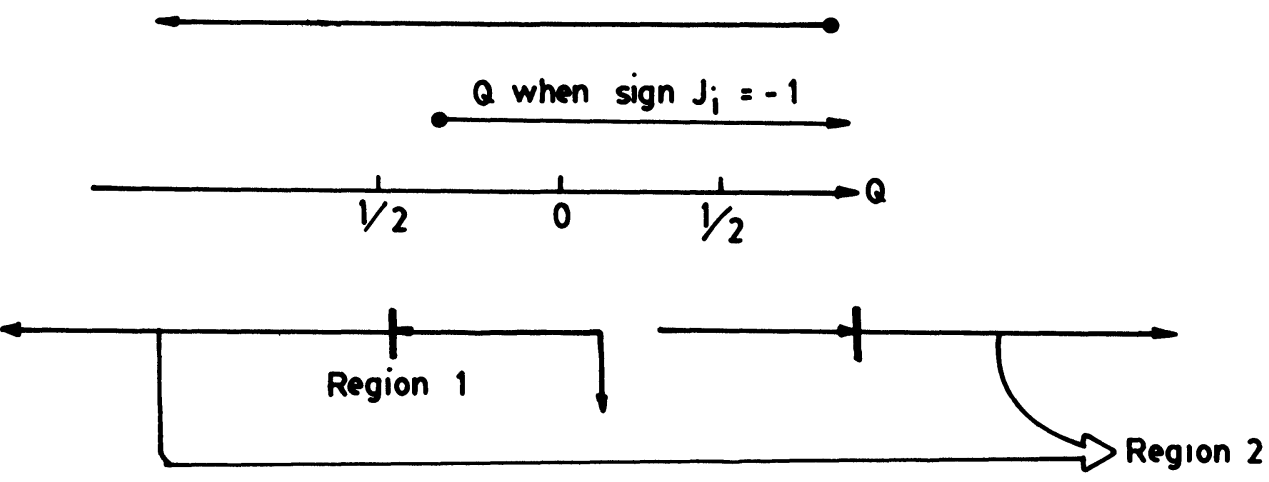

FIGURE A

CASE $1-$ when sign $\hat{\mathrm{J}}_{\mathrm{i}}=+1$, i.e., $\dot{\mathrm{X}}_{2}$ leads $\dot{\mathrm{X}}_{1}$ then $\mathrm{Q}>-1 / 2$.

CASE II-when sign $\mathrm{J}_{\mathrm{i}}=-1$, i.e., $\dot{\mathrm{X}}_{2}$ lags $\dot{\mathrm{X}}_{1} \mathrm{Q}$ when Sign $\hat{\mathrm{J}}_{\mathrm{i}}=+1$ then $\mathrm{Q}<$ $-1 / 2$.

From Fig. (A), it is clear that there are two modes of dynamic behavior that could be implied by the condition of equation (20). In one of the modes, (that is the Region -1 in Fig. (A))

$$
\left|\frac{1-\frac{\mathbf{J}_{12} \mathbf{J}_{21}}{\mathbf{J}_{1} \mathbf{J}_{2}}}{\frac{\mathbf{J}_{21}}{\mathbf{J}_{2}}-\frac{\mathbf{J}_{12}}{\mathbf{J}_{1}}}\right|<1 / 2
$$

A fulfilment of condition (21) implies that condition (20) is satisfied. Condition (20), however, is satisfied in this region for both values of $\operatorname{Sign}\left(\hat{\mathrm{J}}_{1}\right)$ positive or negative. Hence, the present theory does not determine for this region whether one of the waves is leading or whether it is lagging the second. Furthermore, the present analysis even permits $\operatorname{Sign}\left(\hat{\mathrm{J}}_{\mathrm{i}}\right)$ to change in time, and as a result according to (16), A is not necessarily a constant when the coupled oscillators approach synchronization and (19) is not strictly correct. Therefore, the range of values of parameters determined by (21) should be excluded from the present treatment as they do not satisfy initial assumptions. An experimental check of the system behavior in this range has shown that the dynamic behavior is different from the one implied by Fig. 3. The waves in this region sometimes appeared to be synchronized, when several periods of one oscillator lasted the same time as one period of the other.

The second mode implied by the condition (20) occurs when (i.e., in the region -2 shown in Fig. (A)), the system satisfies the following condition:

$$
\left|\frac{1-\frac{J_{12} J_{21}}{J_{1} J_{2}}}{\frac{J_{21}}{J_{2}}-\frac{J_{12}}{J_{1}}}\right|>1 / 2
$$


It can be shown that a sufficient condition to satisfy (22) is that

$\frac{\mathbf{J}_{12} \mathbf{J}_{21}}{\mathbf{J}_{1} \mathbf{J}_{2}}>1$

Hence, in this range of dynamic behavior, $\operatorname{Sign}\left(\hat{\mathrm{J}}_{\mathrm{i}}\right)$ is relatively simply determined by the system parameters, namely

$$
\left(\frac{\mathbf{J}_{21}}{\mathbf{J}_{2}}-\frac{\mathbf{J}_{12}}{\mathbf{J}_{1}}\right) \operatorname{Sign}\left(\hat{\mathbf{J}}_{\mathrm{i}}\right)<0
$$

If $\operatorname{Sign}\left(\hat{\mathrm{J}}_{\mathrm{i}}\right)$ is such that condition (24) would be satisfied then the coupled oscillators approach synchronization, and $\operatorname{Sign}\left(\hat{\mathrm{J}}_{\mathrm{i}}\right)$ does not change from one half cycle to another. If $\operatorname{Sign}\left(\hat{\mathrm{J}}_{\mathrm{i}}\right)$ is initially such that condition $(24)$ is not satisfied, then according to equations (14) and (19), the absolute value of the difference between the two periods, $\left|D T_{i}\right|$, will increase. Each successive half cycle causes a greater difference between the two periods until $\hat{J}_{1}$ changes its sign. Now the inequality (20) is satisfied. Hence, the transient response $J_{0}^{1}(A-1 / A)$ approaches zero (when $\mathrm{i} \rightarrow \infty$ ) and the periods, of both of the oscillators approach the same steady-state period $\mathrm{T}$, which can be evaluated by (17). The mutual position of the two waves (leading or lagging) also possess a steady-state value $\hat{\mathrm{J}}_{\mathrm{i}}$ which is calculated by the approach of $\hat{\mathrm{J}}_{\mathrm{i}}$ to final when $\mathrm{i} \rightarrow \infty$. This is done by substituting $\mathrm{T}$ in equation (10) or equation (11), and, with the aid of (24), one obtains an expression for the steadystate value of phase difference.

$|\hat{\mathbf{J}}|=K \mathbf{J}_{12} J_{21} \frac{\frac{1}{J_{12}}-\frac{1}{J_{21}}-\frac{1}{J_{2}}+\frac{1}{J_{1}}}{J_{21} / J_{2}-J_{12} / J_{1}}$

where the sign negative or positive is determined by inequality (24). A positive $\hat{\mathbf{J}}$ would mean that the second oscillator leads the first while a negative $\hat{J}$ would mean that the second oscillator lags the first. Another implication of (24) is that the present theory is valid only if the evaluation of (25) yields a nonnegative number.

The present analysis has assumed that $|\hat{\mathrm{J}}| \leqslant \mathrm{T} / 2$. If this condition is not satisfied, synchronization would not be obtained. The borders of stable and unstable regions $=0$, and $\pm T / 2$ are singular points.

Hence, the main objective of this section, which was to evaluate the final time period, the entrained frequency of oscillations, and the final phase shift between the two waves has been calculated with a very simple analytical treatment without resorting to approximations. The equations (17) and (25) give the exact expressions for the final time period and the final phase shift respectively.

\section{EXPERIMENTAL RESULTS OF COUPLED MULTIVIBRATORS}

The coupled system in Fig. 4 has been operated with different coupling resistors. The results are in agreement with the presented theory. The experimental results 
here are due to the main mode of dynamic behavior that occurs when the values of the parameters satisfy (22).

Fig. (A) shows the system waveforms when $R_{12}=20 \mathrm{~K} \Omega$ and $R_{21} 30 \mathrm{~K} \Omega$. The intrinsic frequencies of the oscillators were evaluated and they possess the values of 142 and $135 \mathrm{~Hz}$, respectively. The measured entrained frequency and phase shift are $124 \mathrm{~Hz}$ and $-103^{\circ}$ respectively. The theoretical entrained frequency and phase shift, evaluated from (17) and (25) are $128 \mathrm{~Hz}-109^{\circ}$ respectively.

Similar results for an opposite phase shift are demonstrated in Fig. (B) $R_{12}=$ $40 \mathrm{~K} \Omega$ and $R_{21}=10 \mathrm{~K} \Omega$ in this experiment. The results for several such experiments are summarized in Table $\mathbf{I}$.

A dynamic behavior of a special interest is expected when both oscillators possess the same intrinsic frequency. Then, $T_{1}=T_{2}$ and (17) results.

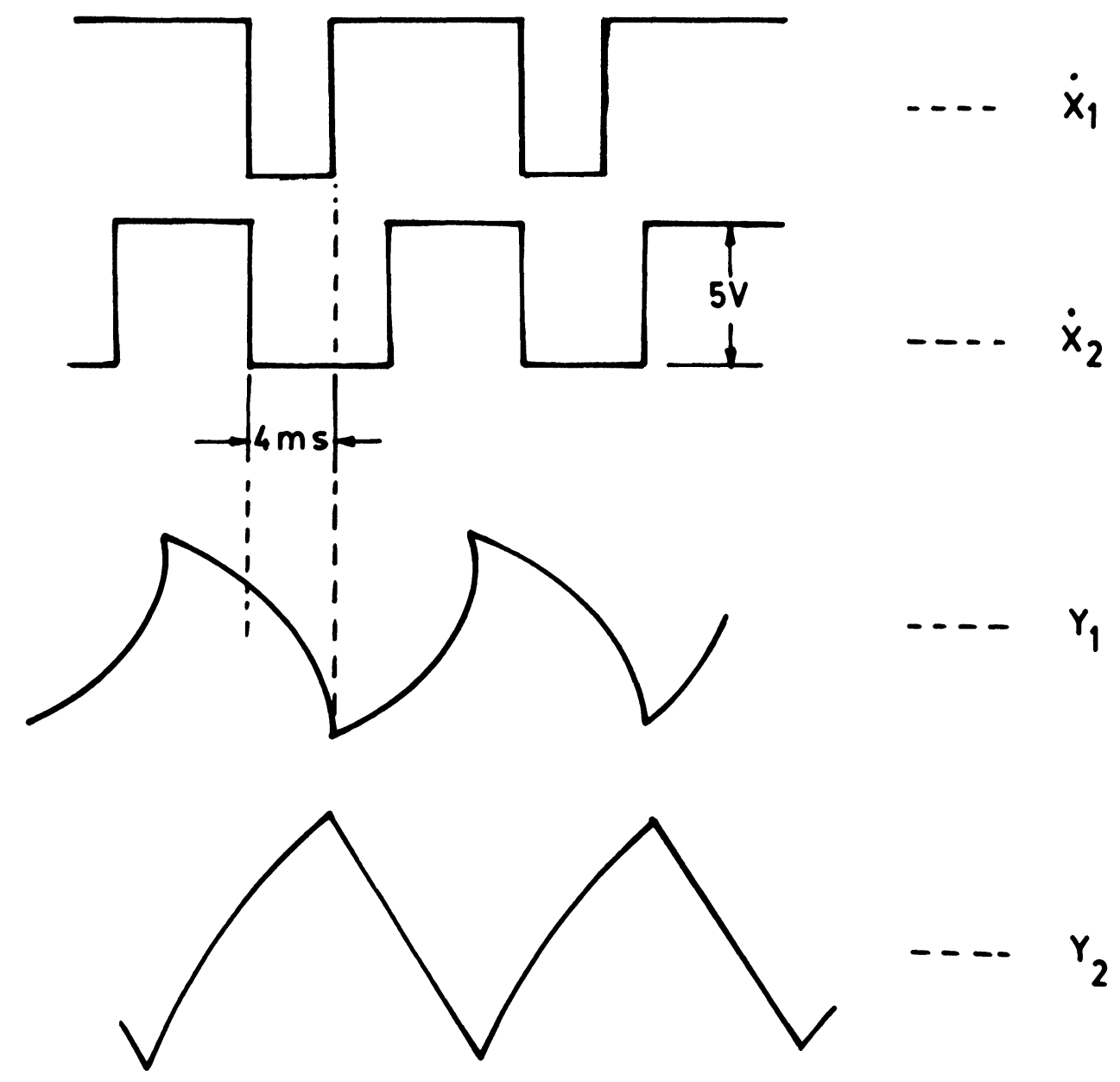

FIGURE A The output signals of the coupled multivibrators system when $R_{12}=20 \mathrm{~K} \Omega$ and $R_{21}=$ $30 \mathrm{~K} \Omega\left(\mathrm{R}_{1}=88 \mathrm{~K} \Omega, \mathrm{R}_{2}=71 \mathrm{~K} \Omega, \mathrm{C}_{1}=10 \mu \mathrm{F}, \mathrm{C}_{2}=1.3 \mu \mathrm{F}, \mathrm{K}=0.2\right)$. 


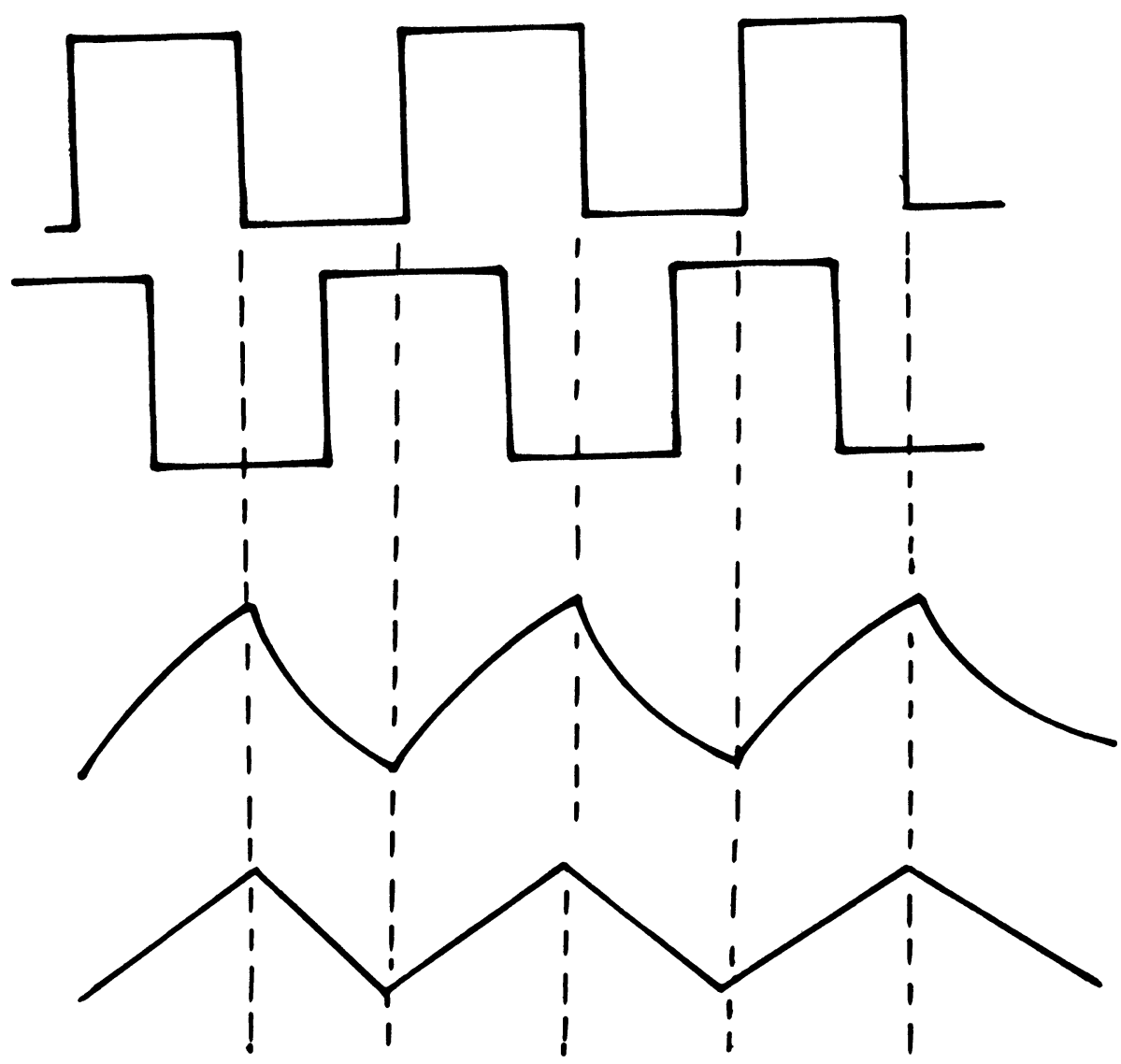

FIGURE B Output signals of coupled multivibrators system when $R_{12}=40 \mathrm{~K} \Omega$ and $R_{21}=10 \mathrm{~K} \Omega$ $\left(R_{1}=8.8 \mathrm{k} \Omega, R_{2}=7.1 \mathrm{~K} \Omega \mathrm{C}_{1}=1.0 \mu \mathrm{F}, \mathrm{C}_{2}=1.3 \mu \mathrm{F}, \mathrm{K}=0.2\right)$. The upper waveform represents the square wave outputs (the upper trace represents $\hat{\mathrm{X}}_{1}$ and the lower trace represents $\hat{\mathrm{X}}_{2}$ ). The lower waveform represents the $\mathrm{Y}$ outputs.

The upper waveform represents the square wave outputs. The lower waveform represents the Y outputs. 741 OpAmps are used.

$\mathrm{T}$ is equal to the common intrinsic period, which is $4 \mathrm{KT}_{1}$. Equation (25) results for $T_{1}=T_{2}$.

$\hat{\mathbf{T}}=-\mathrm{KT}_{1} \operatorname{Sign}\left(\mathrm{J}_{21}-\mathbf{J}_{12}\right)$

TABLE I

Experimental and evaluated results of entrained frequency and phase for various choice of coupling resistors in the system of fig.

\begin{tabular}{|c|c|c|c|c|c|}
\hline \multirow[t]{2}{*}{$R_{12} K \Omega$} & \multirow[t]{2}{*}{$R_{21} K \Omega$} & \multicolumn{2}{|c|}{ Evaluated results } & \multicolumn{2}{|c|}{ Experimental results } \\
\hline & & Frequency $(\mathrm{Hz})$ & Phase $0^{\circ}$ & Frequency $(\mathrm{Hz})$ & Phase $0^{\circ}$ \\
\hline 30 & 30 & 111 & -152 & 104 & 165 \\
\hline 40 & 10 & 145 & 81 & 147 & 79 \\
\hline 20 & 30 & 128 & -109 & 124 & -103 \\
\hline
\end{tabular}


from (26)

$=-\mathrm{T} / 4 \operatorname{Sign}\left(\mathrm{T}_{21}-\mathrm{T}_{12}\right)$

which means that the phase shift between the oscillations is equivalent to $90^{\circ}$ and does not depend on the value of $T_{12}$ and $T_{21}$. The sign of the phase shift depends on these values. An experiment was successfully conducted and verified for the values of $R_{1}, R_{2}, C_{1}$, such that $R_{1} C_{1}=R_{2} C_{2}$. The parameters chosen are such that $T_{12} / T_{1}>1$ and $T_{21} / T_{2}>1$. This ensures that (22) is satisfied. $K$ was always chosen as $0 \cdot 2$ in both oscillators in this series of experiments. It has been observed that even for large coupling resistors of values of about $3 \mathrm{M} \Omega$, the system preserves its expectedly quadrature mode of operation predicted in (27).

\section{CONCLUSION}

The characteristics of the main synchronization state of two coupled multivibrators have been shown to depend on the multivibrators phase shift. The analytical solution of the coupled system dynamics depend on difference equation (15)

$$
A \Delta T_{i}^{1}+T_{i}^{1}=T
$$

where the independent variable $i$ is a running integer number that counts the sequence, in time, of the system generated half cycle. It has also been observed that the system may exhibit modes of dynamic behavior that do not lead to synchronization. This is not extraordinary. In the mode that leads to a synchronized steady state, the expressions for the final entrained frequency and final phase shift are given below in terms of system parameters,

$F=\frac{1}{T}=\frac{J_{21} / J_{2}-J_{12} / J_{1}}{4 K\left(J_{21}-J_{12}\right)}$

$\mathbf{J}_{1}=\mathrm{R}_{1} \mathrm{C}_{1} ; \mathrm{J}_{12}=\mathrm{R}_{12} \mathrm{C}_{1} ; \mathrm{J}_{2}=\mathrm{RC}_{2}, \mathrm{~J}_{21}=\mathrm{RC}_{2}, \mathrm{~K}=\mathrm{R}_{\mathrm{a}} / \mathrm{R}_{\mathrm{b}}$. These expressions are valid if inequality (22) is satisfied.

An important feature of the present work is that the results (i.e., expression for final frequency and phase shift) have been obtained by completely analytical method, without resorting to approximations.

The quasi-chaotic behavior observed on the oscilloscope is due to the fact that in the present system, both multivibrators possess a flexible behavior that allows both of them to change the frequency by the effect of the other multivibrator. Hence, chaotic behavior is less likely.

The relationship between the system of sinusoidal coupled oscillators and the system of coupled multivibrators is due to similarity of structures. The structural similarity between the two models results in a related dynamic behavior in both cases. The stability regions, for example, and also the singular points, are closely related. In the system of two sinusoidal oscillators, one phase shift results in a 
stable steady synchronization state, while the synchronization characterized by the phase of opposite sign is unstable, although it is still a steady-state of the system, and similar results have been obtained in the system of the two coupled multivibrators.

One of the reasons for the similarity between the two systems of oscillators is that, in a multivibrator system, the amplitude of the square waves is determined by the level of saturation of the hysteritic comparator and, hence, the waves are stabilized in size, which is somewhat similar to the amplitudes of the two waves of the sinusoidal system.

The integrated signal in each multivibrator merely affects the frequency. Its effect on the neighbor's frequency is larger; the larger is its signal compared with that of the neighbor, since the sum of both signals is integrated in the multivibrator integrator. The sum is larger and the integration time is shorter because the multivibrator reaches saturation sooner and then changes to another state. The effect on phase shift is also similar. Since the larger is the coincidence in time between the waves the shorter is the integration time before saturation is reached. Hence, the smaller is the phase shift between multivibrators, the larger is the effect of one waves on the frequency of the other.

This paper deals with the dynamic behavior and synchronization of a two coupled multivibrator. Other modes of dynamic behavior have not been analysed. Furthermore, a thorough treatment is given merely to a mode of synchronization that is regarded as the main mode of synchronization. This mode will govern the system if inequality (22) is satisfied. The parameters chosen for most of the experimental work are such that both $J_{12} / J_{1}>1$ and $J_{21} / J_{2}>1$. This assures that inequality (22) is satisfied. We also believe that this range of parameters is more likely to represent natural systems, since it implies relatively small couplings between the multivibrators.

\section{REFERENCES}

1. Theo Pavlidis, Biological Oscillators: Their Mathematical Analysis, New York: Academic, 1973.

2. D.A. Linkens, "Stability of entrainment conditions for a particular form of mutually coupled Van der Pol Oscillators," IEEE Trans. Circuits and Systems, Vol. CAS-23, pp. 113-121, 1976.

3. B.Z. Kaplan and K. Radoanvar, "Canonic coupling of sinusoidal oscillators," Proc. IEEE, Vol. 70. pp. 1130-1132, 1982.

4. B.C. Goodwin, Analytical Physiology of Cells and Developing organisms, Academic, London, England, 1976.

5. E. Palm and M. Tvcitercid, "On coupled Van der Pol equations," Quart. J. Mechanics Appl. Maths, Vol. 33, pt. 3, pp. 267-276, 1980.

6. L.D. Harmon, "Neuromimes: Action of a reciprocally inhibitory pair," Science, Vol. 146, pp. 1323-1325, 1964.

7. C.K. Kohli et al, "Ilysterctic system for neural-type circuits," Proc. IEEE, Vol. 69, pp. 285-287, 1982.

8. U. Tietze and Ch. Sehenk, Advanced Electronic Circuits, Springer, Berlin, Germany, 1978, pp. 242-248.

9. B.Z. Kaplan and K. Radparvar, "Canonic coupling of sinusoidal oscillators: New Results," to be published.

10. Y.S. Tang, A.I. Mees and Leon O. Chua, "Synchronization and Chaos," IEEE Trans. Circuits and Systems, Vol. CAS-30, pp. 620-626, 1983. 

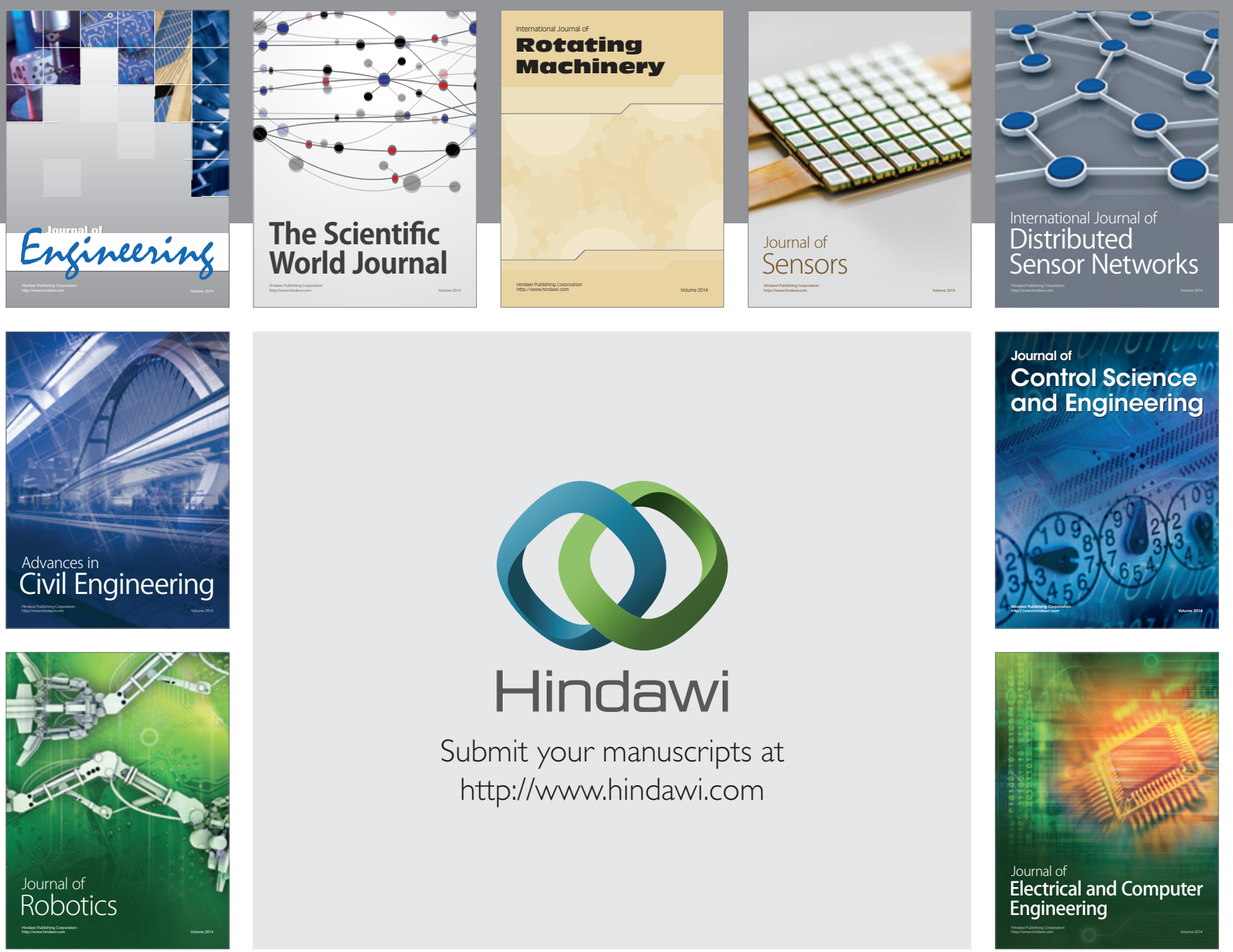

Submit your manuscripts at

http://www.hindawi.com
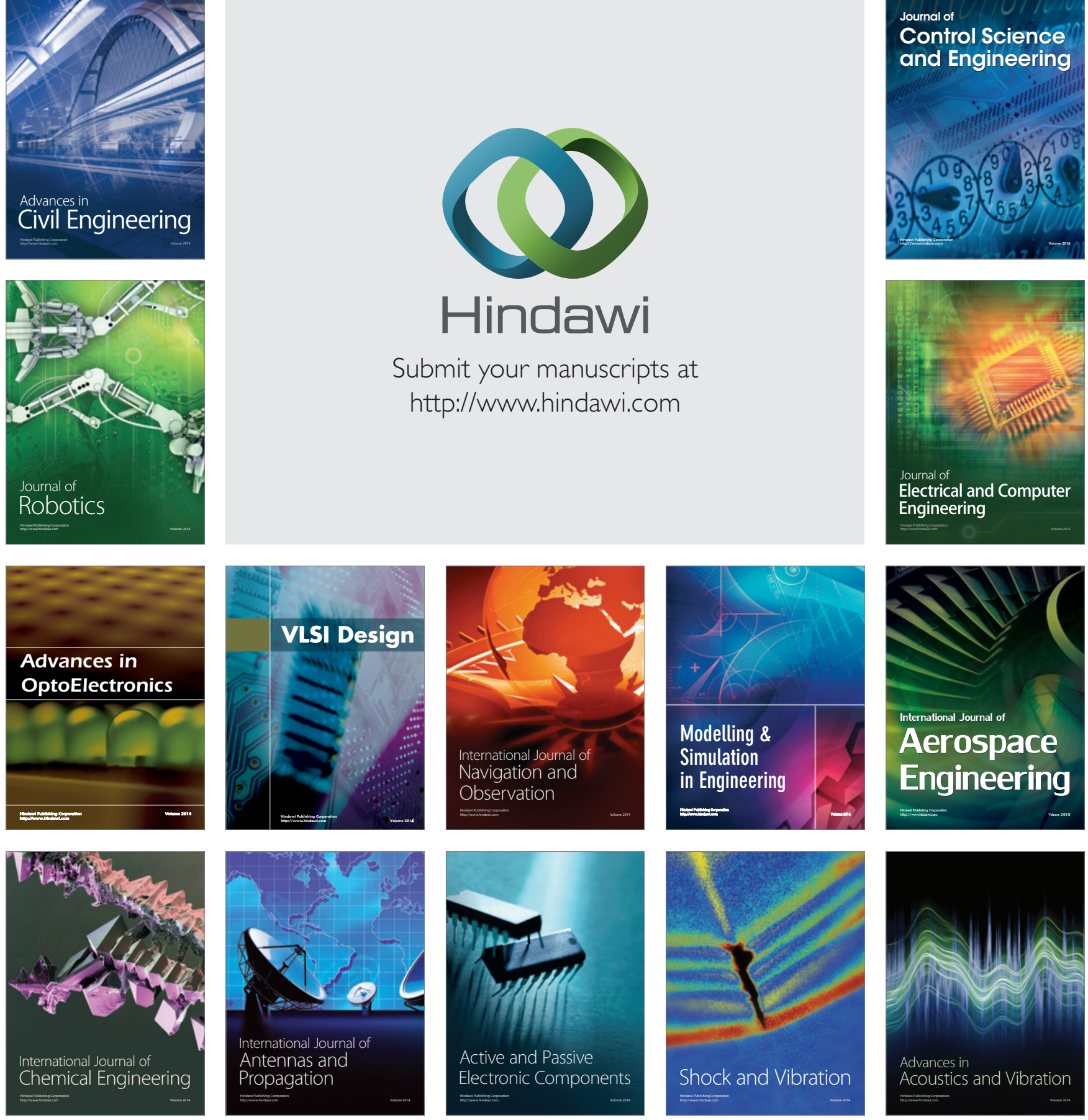